\title{
Analysis and applications of the proportional Caputo derivative
}

\author{
Ali Akgül ${ }^{1 *}$ (D) and Dumitru Baleanu $u^{2,3}$
}

\section{"Correspondence:}

aliakgul00727@gmail.com

${ }^{1}$ Art and Science Faculty,

Department of Mathematics, Siirt

University, TR-56100 Siirt, Turkey

Full list of author information is

available at the end of the article

\begin{abstract}
In this paper, we investigate the analysis of the proportional Caputo derivative that recently has been constructed. We create some useful relations between this new derivative and beta function. We discretize the new derivative. We investigate the stability and obtain a stability condition for the new derivative.
\end{abstract}

\section{Introduction}

Fractional calculus is an emerging field of mathematics [1] having important contributions in modeling the dynamics of complex systems $[2,3]$ from various fields of science and engineering $[4,5]$. Nowadays a huge debate was opened by asking the simple" question: can we classify the fractional operators?" Curiously the answer of this question is not simple and, so far, several answers seemed to be possible [6-11]. A new non-singular fractional operator was proposed by Caputo and Fabrizio [12] and their result was generalized by Atangana and Baleanu [13] and applied successfully to a lot of complex phenomena including biological ones.

Khalid et al. [14] have studied the computational research of the Caputo time fractional Allen-Cahn equation. Owolabi [15] has studied by analysis and numerical simulation a multicomponent system with the Atangana-Baleanu fractional derivative. Akgül [16] has presented a novel method for a fractional derivative with non-local and non-singular kernel. Akgül [17] has investigated the solutions of differential equations with the generalized fractional derivatives. Atangana et al. [18] have investigated the analysis of the fractal fractional derivatives in detail. Fernandez et al. [19] investigated the series representations for fractional-calculus operators involving generalized Mittag-Leffler functions. Wu et al. [20] have investigated the fractional impulsive differential equations including the exact solutions, integral equations and short memory case. Some inequalities were investigated within the proportional fractional operators [21,22] and in [23] was investigated the proportional derivatives of a function with respect to another function. Very recently, a new fractional operator has been constructed in [24]:

$$
{ }_{0}^{P C} D_{t}^{\alpha} f(t)=\frac{1}{\Gamma(1-\alpha)} \int_{0}^{t}\left(k_{1}(\alpha, \tau) f(\tau)+k_{0}(\alpha, \tau) \frac{d f(\tau)}{d \tau}\right)(t-\tau)^{-\alpha} d \tau .
$$

(c) The Author(s) 2021. This article is licensed under a Creative Commons Attribution 4.0 International License, which permits use, sharing, adaptation, distribution and reproduction in any medium or format, as long as you give appropriate credit to the original author(s) and the source, provide a link to the Creative Commons licence, and indicate if changes were made. The images or other third party material in this article are included in the article's Creative Commons licence, unless indicated otherwise in a credit line to the material. If material is not included in the article's Creative Commons licence and your intended use is not permitted by statutory regulation or exceeds the permitted use, you will need to obtain permission directly from the copyright holder. To view a copy of this licence, visit http://creativecommons.org/licenses/by/4.0/. 
In this paper, we aim to analyze the above derivative in detail for $k_{0}(\alpha, t)=\left(\alpha t^{1-\alpha}\right) c^{2 \alpha}$ and $k_{1}(\alpha, t)=(1-\alpha) t^{\alpha}$. Here $c$ is as a constant of the time dimension $t$ for the two terms involved in the new derivative (1.1).

The new fractional operator in the Caputo sense is a generalization of the classical proportional derivative introduced by [24] which has deep applications in control theory. The new fractional operator will provide better applications in control theory. Due to the physical meaning of the initial conditions we concentrate here on the Caputo fractional generalization. For more details see [25-28].

We construct the paper as follows. We give some scientific theorems for the new derivative in Sect. 2. We present the discretization and the applications of the proportional Caputo derivative in Sect. 3. We show the stability analysis in Sect. 4. We demonstrate the numerical results in Sect. 5. We discuss the conclusion in the last section.

\section{Analysis of the proportional Caputo derivative}

We present the following scientific results for the new derivative.

Lemma 2.1 We have the following relation for the new derivative given by (1.1):

$$
\begin{aligned}
\left|{ }_{0}^{P C} D_{t}^{\alpha} f(t)\right|< & \frac{t(1-\alpha)}{\Gamma(1-\alpha)}\|f(\tau)\|_{\infty} B(\alpha+1,1-\alpha) \\
& +\frac{\alpha c^{2 \alpha}}{\Gamma(1-\alpha)} t^{2-2 \alpha}\left\|\frac{d f(\tau)}{d \tau}\right\|_{\infty} B(2-\alpha, 1-\alpha) .
\end{aligned}
$$

Proof We have

$$
\begin{aligned}
\left|{ }_{0}^{P C} D_{t}^{\alpha} f(t)\right|= & \frac{1}{\Gamma(1-\alpha)}\left|\int_{0}^{t}\left((1-\alpha) \tau^{\alpha} f(\tau)+c^{2 \alpha} \alpha \tau^{1-\alpha} \frac{d f(\tau)}{d \tau}\right)(t-\tau)^{-\alpha} d \tau\right| \\
\leq & \frac{1}{\Gamma(1-\alpha)}\left|\int_{0}^{t}\left((1-\alpha) \tau^{\alpha} f(\tau)\right)(t-\tau)^{-\alpha} d \tau\right| \\
& +\frac{c^{2 \alpha}}{\Gamma(1-\alpha)}\left|\int_{0}^{t}\left(\alpha \tau^{1-\alpha} \frac{d f(\tau)}{d \tau}\right)(t-\tau)^{-\alpha} d \tau\right| \\
< & \frac{1}{\Gamma(1-\alpha)} \int_{0}^{t}|f(\tau)|(1-\alpha) \tau^{\alpha}(t-\tau)^{-\alpha} d \tau \\
& +\frac{c^{2 \alpha}}{\Gamma(1-\alpha)} \int_{0}^{t}\left|\frac{d f(\tau)}{d \tau}\right| \alpha \tau^{1-\alpha}(t-\tau)^{-\alpha} d \tau \\
< & \frac{1}{\Gamma(1-\alpha)} \int_{0}^{t} \sup _{\tau \in[0, t]}|f(\tau)|(1-\alpha) \tau^{\alpha}(t-\tau)^{-\alpha} d \tau \\
& +\frac{c^{2 \alpha}}{\Gamma(1-\alpha)} \int_{0}^{t} \sup _{\tau \in[0, t]}\left|\frac{d f(\tau)}{d \tau}\right| \alpha \tau^{1-\alpha}(t-\tau)^{-\alpha} d \tau .
\end{aligned}
$$

Then we obtain

$$
\begin{aligned}
\left.\right|_{0} ^{P C} D_{t}^{\alpha} f(t) \mid< & \frac{(1-\alpha)}{\Gamma(1-\alpha)}\|f(\tau)\|_{\infty} \int_{0}^{t} \tau^{\alpha}(t-\tau)^{-\alpha} d \tau \\
& +\frac{\alpha c^{2 \alpha}}{\Gamma(1-\alpha)}\left\|\frac{d f(\tau)}{d \tau}\right\|_{\infty} \int_{0}^{t} \tau^{1-\alpha}(t-\tau)^{-\alpha} d \tau
\end{aligned}
$$


Let $\tau=t h$. Then we obtain

$$
\begin{aligned}
\left|{ }_{0}^{P C} D_{t}^{\alpha} f(t)\right|< & \frac{(1-\alpha)}{\Gamma(1-\alpha)}\|f(\tau)\|_{\infty} \int_{0}^{1}(t h)^{\alpha}(t-t h)^{-\alpha} t d h \\
& +\frac{\alpha c^{2 \alpha}}{\Gamma(1-\alpha)}\left\|\frac{d f(\tau)}{d \tau}\right\|_{\infty} \int_{0}^{1}(t h)^{1-\alpha}(t-t h)^{-\alpha} t d h \\
< & \frac{t(1-\alpha)}{\Gamma(1-\alpha)}\|f(\tau)\|_{\infty} \int_{0}^{1} h^{\alpha}(1-h)^{-\alpha} d h \\
& +\frac{\alpha c^{2 \alpha}}{\Gamma(1-\alpha)} t^{2-2 \alpha}\left\|\frac{d f(\tau)}{d \tau}\right\|_{\infty} \int_{0}^{1} h^{1-\alpha}(1-h)^{-\alpha} d h .
\end{aligned}
$$

Then we get the desired result:

$$
\begin{aligned}
\left|{ }_{0}^{P C} D_{t}^{\alpha} f(t)\right|< & \frac{t(1-\alpha)}{\Gamma(1-\alpha)}\|f(\tau)\|_{\infty} B(\alpha+1,1-\alpha) \\
& +\frac{\alpha c^{2 \alpha}}{\Gamma(1-\alpha)} t^{2-2 \alpha}\left\|\frac{d f(\tau)}{d \tau}\right\|_{\infty} B(2-\alpha, 1-\alpha) .
\end{aligned}
$$

This completes the proof.

Remark 2.2 We consider

$$
\begin{aligned}
& { }_{0}^{P C} D_{x}^{\gamma}(u(x) v(x)) \\
& \quad=\frac{1}{\Gamma(1-\gamma)} \int_{0}^{x}\left((1-\gamma) t^{\gamma} u(t) v(t)+\gamma c^{2 \gamma} t^{1-\gamma} \frac{d u(t) v(t)}{d t}\right)(x-t)^{-\gamma} d t .
\end{aligned}
$$

If $u$ and $v$ are continuous and bounded, then we get

$$
\begin{aligned}
{ }_{0}^{P C} D_{x}^{\alpha}(u(x) v(x))= & \frac{1}{\Gamma(1-\gamma)} \int_{0}^{x}\left((1-\gamma) t^{\gamma} u(t) v(t)\right)(x-t)^{-\gamma} d t \\
& +\frac{1}{\Gamma(1-\gamma)} \int_{0}^{x} \gamma c^{2 \gamma} t^{1-\gamma}\left(\frac{d u(t)}{d t} v(t)+\frac{d \nu(t)}{d t} u(t)\right)(x-t)^{-\gamma} d t .
\end{aligned}
$$

Lemma 2.3 Assume that $f$ and $g$ are differentiable and bounded. Then we obtain

$$
\begin{aligned}
\left|{ }_{0}^{P C} D_{t}^{\alpha}(f(t) g(t))\right|< & \frac{t(1-\alpha)}{\Gamma(1-\alpha)}\|f(t)\|_{\infty}\|g(t)\|_{\infty} B(\alpha+1,1-\alpha) \\
& +\frac{\alpha t^{2-2 \alpha}}{\Gamma(1-\alpha)}\left\|\frac{d f(t)}{d t}\right\|_{\infty}\|g(t)\|_{\infty} B(2-\alpha, 1-\alpha) \\
& +\frac{\alpha t^{2-2 \alpha}}{\Gamma(1-\alpha)}\left\|\frac{d g(t)}{d t}\right\|_{\infty}\|f(t)\|_{\infty} B(2-\alpha, 1-\alpha) .
\end{aligned}
$$


Proof We have

$$
\begin{aligned}
\left|{ }_{0}^{P C} D_{t}^{\alpha}(f(t) g(t))\right|< & \frac{t(1-\alpha)}{\Gamma(1-\alpha)}\|f(t)\|_{\infty}\|g(t)\|_{\infty} B(\alpha+1,1-\alpha) \\
& +\frac{\alpha t^{2-2 \alpha}}{\Gamma(1-\alpha)}\left\|\frac{d f(t)}{d t}\right\|_{\infty}\|g(t)\|_{\infty} B(2-\alpha, 1-\alpha) \\
& +\frac{\alpha t^{2-2 \alpha}}{\Gamma(1-\alpha)}\left\|\frac{d g(t)}{d t}\right\|_{\infty}\|f(t)\|_{\infty} B(2-\alpha, 1-\alpha) .
\end{aligned}
$$

Let $\tau=t h$. Then we obtain

$$
\begin{aligned}
\left|{ }_{0}^{P C} D_{t}^{\alpha}(f(t) g(t))\right|< & \frac{t(1-\alpha)}{\Gamma(1-\alpha)}\|f(t)\|_{\infty}\|g(t)\|_{\infty} B(\alpha+1,1-\alpha) \\
& +\frac{\alpha t^{2-2 \alpha}}{\Gamma(1-\alpha)}\left\|\frac{d f(t)}{d t}\right\|_{\infty}\|g(t)\|_{\infty} B(2-\alpha, 1-\alpha) \\
& +\frac{\alpha t^{2-2 \alpha}}{\Gamma(1-\alpha)}\left\|\frac{d g(t)}{d t}\right\|_{\infty}\|f(t)\|_{\infty} B(2-\alpha, 1-\alpha) .
\end{aligned}
$$

This completes the proof.

Lemma 2.4 Iff and $g$ are differentiable and satisfy the following condition:

$$
\left\|\frac{d f}{d t}-\frac{d g}{d t}\right\|_{\infty}<K\|f-g\|_{\infty}
$$

then we have

$$
\left\|{ }_{0}^{P C} D_{t}^{\alpha} f(t)-{ }_{0}^{P C} D_{t}^{\alpha} g(t)\right\|_{\infty}<K\|f-g\|_{\infty} .
$$

Proof We have

$$
\begin{aligned}
\left\|_{0}^{P C} D_{t}^{\alpha} f(t)-{ }_{0}^{P C} D_{t}^{\alpha} g(t)\right\|_{\infty}< & \frac{(1-\alpha)}{\Gamma(1-\alpha)}\|f(t)-g(t)\|_{\infty} \int_{0}^{t} \tau^{\alpha}(t-\tau)^{-\alpha} d \tau \\
& +\frac{\alpha c^{2 \alpha}}{\Gamma(1-\alpha)}\left\|\frac{d f(t)}{d t}-\frac{d g(t)}{d t}\right\|_{\infty} \int_{0}^{t} \tau^{1-\alpha}(t-\tau)^{-\alpha} d \tau .
\end{aligned}
$$

Let $\tau=t h$. Then we obtain

$$
\left\|{ }_{0}^{P C} D_{t}^{\alpha} f(t)-{ }_{0}^{P C} D_{t}^{\alpha} g(t)\right\|_{\infty}<K\|f(t)-g(t)\|_{\infty} .
$$

This completes the proof.

Lemma 2.5 Let $f$ be analytic around 0 , then we obtain

$$
\begin{aligned}
{ }_{0}^{P C} D_{t}^{\alpha} f(t)= & t(1-\alpha) \sum_{j=0}^{\infty} a_{j} t^{j} \frac{\Gamma(j+\alpha+1)}{\Gamma(j+2)} \\
& +t^{1-2 \alpha} \alpha c^{2 \alpha} \sum_{j=0}^{\infty} j a_{j} t^{j} \frac{\Gamma(j-\alpha+1)}{\Gamma(j-2 \alpha+2)} .
\end{aligned}
$$


Proof We have

$$
\begin{aligned}
{ }_{0}^{P C} D_{t}^{\alpha} f(t)= & \frac{1}{\Gamma(1-\alpha)} \int_{0}^{t}\left((1-\alpha) \tau^{\alpha} f(\tau)+\alpha c^{2 \alpha} \tau^{1+\alpha} \frac{d f(\tau)}{d \tau}\right)(t-\tau)^{-\alpha} d \tau \\
= & \frac{(1-\alpha)}{\Gamma(1-\alpha)} \sum_{j=0}^{\infty} a_{j} \int_{0}^{t} \tau^{\alpha+j}(t-\tau)^{-\alpha} d \tau \\
& +\frac{\alpha c^{2 \alpha}}{\Gamma(1-\alpha)} \sum_{j=0}^{\infty} j a_{j} \int_{0}^{t} \tau^{j+\alpha}(t-\tau)^{-\alpha} d \tau .
\end{aligned}
$$

We let $\tau=h t$. Then we obtain

$$
\begin{aligned}
{ }_{0}^{P C} D_{t}^{\alpha} f(t)= & t(1-\alpha) \sum_{j=0}^{\infty} a_{j} t^{\frac{\Gamma(j+\alpha+1)}{\Gamma(j+2)}} \\
& +t^{1-2 \alpha} \alpha c^{2 \alpha} \sum_{j=0}^{\infty} j a_{j} t \frac{\Gamma(j-\alpha+1)}{\Gamma(j-2 \alpha+2)}
\end{aligned}
$$

This completes the proof.

\section{Discretization and applications of the proportional Caputo derivative}

We consider the new derivative [24]:

$$
{ }_{0}^{P C} D_{t}^{\alpha} f(t)=\frac{1}{\Gamma(1-\alpha)} \int_{0}^{t}\left((1-\alpha) \tau^{\alpha} f(\tau)+\alpha c^{2 \alpha} \tau^{1-\alpha} \frac{d f(\tau)}{d \tau}\right)(t-\tau)^{-\alpha} d \tau .
$$

We put $t_{n}=n \Delta t$, then at $t_{n+1}$, we have

$$
\begin{aligned}
{ }_{0}^{P C} D_{t}^{\alpha} f\left(t_{n+1}\right)= & \frac{1}{\Gamma(1-\alpha)} \int_{0}^{t_{n+1}}\left((1-\alpha) \tau^{\alpha} f(\tau)+\alpha c^{2 \alpha} \tau^{1-\alpha} \frac{d f(\tau)}{d \tau}\right)\left(t_{n+1}-\tau\right)^{-\alpha} d \tau \\
= & \frac{1}{\Gamma(1-\alpha)} \sum_{j=0}^{n} \int_{t_{j}}^{t_{j+1}}\left((1-\alpha) t_{j}^{\alpha} f^{j+1}+\alpha c^{2 \alpha} t_{j}^{1-\alpha} \frac{f^{j+1}-f^{j}}{\Delta t}\right)\left(t_{n+1}-\tau\right)^{-\alpha} d \tau \\
= & \frac{1}{\Gamma(1-\alpha)} \sum_{j=0}^{n}\left((1-\alpha) t_{j}^{\alpha} f^{j+1}+\alpha c^{2 \alpha} t_{j}^{1-\alpha} \frac{f^{j+1}-f^{j}}{\Delta t}\right) \\
& \times \int_{t_{j}}^{t_{j+1}}\left(t_{n+1}-\tau\right)^{-\alpha} d \tau \\
= & \frac{1}{\Gamma(1-\alpha)} \sum_{j=0}^{n}\left((1-\alpha) t_{j}^{\alpha} f^{j+1}+\alpha c^{2 \alpha} t_{j}^{1-\alpha} \frac{f^{j+1}-f^{j}}{\Delta t}\right) \\
& \times\left[(n-j+1)^{1-\alpha}-(n-j)^{1-\alpha}\right] .
\end{aligned}
$$

We take into consideration [18]

$$
{ }_{0}^{P C} D_{t}^{\alpha} u(x, t)=f(x, t, u(x, t)) .
$$


Here $u(x, 0)=g(x), x_{m}-x_{m-1}=\Delta x, t_{n+1}-t_{n}=\Delta t, t_{n}=n \Delta t, x_{m}=m \Delta x$. The above equation can be approximated as

$$
\begin{aligned}
& \frac{1}{\Gamma(1-\alpha)} \sum_{j=0}^{n}\left((1-\alpha) t_{j}^{\alpha} u_{m}^{j+1}+\alpha c^{2 \alpha} t_{j}^{1-\alpha} \frac{u_{m}^{j+1}-u_{m}^{j}}{\Delta t}\right)\left[(n-j+1)^{1-\alpha}-(n-j)^{1-\alpha}\right] \\
& \quad=f\left(x_{m}, t_{n+1}, u_{m}^{n+1}\right) .
\end{aligned}
$$

\section{Stability analysis}

We discretize the following problem and investigate the stability of it. We consider the heat equation,

$$
\frac{\partial u(x, t)}{\partial t}=k \frac{\partial^{2} u(x, t)}{\partial x^{2}}
$$

We change the left hand side of the above equation with the new derivative and we obtain

$$
{ }_{0}^{P C} D_{t}^{\alpha} u(x, t)=k \frac{\partial^{2} u(x, t)}{\partial x^{2}}
$$

We obtain

$$
\begin{aligned}
& \frac{1}{\Gamma(1-\alpha)} \sum_{p=0}^{s}\left((1-\alpha) t_{p}^{\alpha} u_{m}^{p+1}+\alpha c^{2 \alpha} t_{p}^{1-\alpha} \frac{u_{m}^{p+1}-u_{m}^{p}}{\Delta t}\right)\left[(s-p+1)^{1-\alpha}-(s-p)^{1-\alpha}\right] \\
& \quad=k \frac{u_{m+1}^{s+1}-2 u_{m}^{s+1}+u_{m-1}^{s+1}}{(\Delta x)^{2}}
\end{aligned}
$$

at $\left(t_{s+1}, x_{m}\right)$. We put $u_{m}^{s}=\delta_{s} \exp \left(i k_{m} x\right)$. Plugging this into the above equation, we obtain

$$
\begin{aligned}
& \frac{1}{\Gamma(1-\alpha)} \sum_{p=0}^{s}\left((1-\alpha) t_{p}^{\alpha} \delta_{p+1} \exp \left(i k_{m} x\right)+\alpha c^{2 \alpha} t_{p}^{1-\alpha} \frac{\delta_{p+1} \exp \left(i k_{m} x\right)-\delta_{p} \exp \left(i k_{m} x\right)}{\Delta t}\right) \\
& \quad \times\left[(s-p+1)^{1-\alpha}-(s-p)^{1-\alpha}\right] \\
& =k \frac{\delta_{s+1} \exp \left(i k_{m}(x+\Delta x)\right)-2 \delta_{s+1} \exp \left(i k_{m} x\right)+\delta_{s+1} \exp \left(i k_{m}(x-\Delta x)\right)}{(\Delta x)^{2}} .
\end{aligned}
$$

After simplification we get

$$
\begin{aligned}
& \frac{1}{\Gamma(1-\alpha)} \sum_{p=0}^{s}\left((1-\alpha) t_{p}^{\alpha} \delta_{p+1}+\alpha c^{2 \alpha} t_{p}^{1-\alpha} \frac{\delta_{p+1}-\delta_{p}}{\Delta t}\right) \\
& \quad \times\left[(s-p+1)^{1-\alpha}-(s-p)^{1-\alpha}\right] \\
& =k \frac{\delta_{s+1} \exp \left(i k_{m}(\Delta x)\right)-2 \delta_{s+1}+\delta_{s+1} \exp \left(i k_{m}(-\Delta x)\right)}{(\Delta x)^{2}} .
\end{aligned}
$$

For simplicity, we take

$$
A_{p, \alpha}=\frac{(1-\alpha)(p \Delta t)^{\alpha}}{\Gamma(1-\alpha)}, \quad B_{p, \alpha}=\frac{\alpha c^{2 \alpha}(p \Delta t)^{1-\alpha}}{\Gamma(1-\alpha) \Delta t}, \quad a=\frac{k}{(\Delta x)^{2}}
$$


Then we obtain

$$
\begin{aligned}
& \sum_{p=0}^{s}\left(A_{p, \alpha} \delta_{p+1}+B_{p, \alpha}\left(\delta_{p+1}-\delta_{p}\right)\right)\left[(s-p+1)^{1-\alpha}-(s-p)^{1-\alpha}\right] \\
& \quad=a \delta_{s+1} \exp \left(i k_{m}(\Delta x)\right)-2 a \delta_{s+1}+a \delta_{s+1} \exp \left(i k_{m}(-\Delta x)\right)
\end{aligned}
$$

Thus, we obtain

$$
\begin{aligned}
& \sum_{p=0}^{s}\left(\left(A_{p, \alpha}+B_{p, \alpha}\right) \delta_{p+1}-B_{p, \alpha} \delta_{p}\right)\left[(s-p+1)^{1-\alpha}-(s-p)^{1-\alpha}\right] \\
& \quad=a \delta_{s+1}\left(\exp \left(i k_{m}(\Delta x)\right)-2+\exp \left(-i k_{m}(\Delta x)\right)\right)
\end{aligned}
$$

Using the relation between the trigonometric functions and exponential functions gives

$$
\sum_{p=0}^{s}\left(\left(A_{p, \alpha}+B_{p, \alpha}\right) \delta_{p+1}-B_{p, \alpha} \delta_{p}\right)\left[(s-p+1)^{1-\alpha}-(s-p)^{1-\alpha}\right]=-4 a \delta_{s+1} \sin ^{2}\left(\frac{k_{m} \Delta x}{2}\right)
$$

For $s=0$, we obtain

$$
\left(\left(A_{0, \alpha}+B_{0, \alpha}\right) \delta_{1}-B_{0, \alpha} \delta_{0}\right)=-4 a \delta_{1} \sin ^{2}\left(\frac{k_{m} \Delta x}{2}\right)
$$

Here $\left|\frac{\delta_{1}}{\delta_{0}}\right|<1$ implies

$$
\left|\frac{B_{0, \alpha}}{A_{0, \alpha}+B_{0, \alpha}+4 a \sin ^{2}\left(\frac{k_{m} \Delta x}{2}\right)}\right|<1 \text {. }
$$

This is true for $\forall m$. Thus, we get

$$
\left|\frac{B_{0, \alpha}}{A_{0, \alpha}+B_{0, \alpha}+4 a}\right|<1
$$

We assume that $\left|\frac{\delta_{s}}{\delta_{0}}\right|<1$. We need to show that $\left|\frac{\delta_{s+1}}{\delta_{0}}\right|<1$. We know that

$$
\sum_{p=0}^{s}\left(\left(A_{p, \alpha}+B_{p, \alpha}\right) \delta_{p+1}-B_{p, \alpha} \delta_{p}\right)\left[(s-p+1)^{1-\alpha}-(s-p)^{1-\alpha}\right]=-4 a \delta_{s+1} \sin ^{2}\left(\frac{k_{m} \Delta x}{2}\right)
$$

Then we get

$$
\left|-4 a \delta_{s+1} \sin ^{2}\left(\frac{k_{m} \Delta x}{2}\right)\right|=\left|\sum_{p=0}^{s}\left(\left(A_{p, \alpha}+B_{p, \alpha}\right) \delta_{p+1}-B_{p, \alpha} \delta_{p}\right)\left[(s-p+1)^{1-\alpha}-(s-p)^{1-\alpha}\right]\right|
$$

Thus, we reach

$$
\delta_{s+1}\left|-4 a \sin ^{2}\left(\frac{k_{m} \Delta x}{2}\right)\right|<\delta_{0}\left|\sum_{p=0}^{s}\left(\left(A_{p, \alpha}+B_{p, \alpha}\right)-B_{p, \alpha}\right)\left[(s-p+1)^{1-\alpha}-(s-p)^{1-\alpha}\right]\right| .
$$


Here $\left|\frac{\delta_{s+1}}{\delta_{0}}\right|<1$ implies

$$
\frac{\sum_{p=0}^{s}\left|\left(\left(A_{p, \alpha}+B_{p, \alpha}\right)-B_{p, \alpha}\right)\left[(s-p+1)^{1-\alpha}-(s-p)^{1-\alpha}\right]\right|}{\left|-4 a \sin ^{2}\left(\frac{k_{m} \Delta x}{2}\right)\right|}<1 .
$$

This is true for $\forall m$. Thus, we get

$$
\frac{\sum_{p=0}^{s}\left|\left(\left(A_{p, \alpha}+B_{p, \alpha}\right)-B_{p, \alpha}\right)\left[(s-p+1)^{1-\alpha}-(s-p)^{1-\alpha}\right]\right|}{|-4 a|}<1
$$

Therefore, the method is stable if

$$
\min \left(\left|\frac{B_{0, \alpha}}{A_{0, \alpha}+B_{0, \alpha}+4 a}\right|, \frac{\sum_{p=0}^{s}\left|\left(\left(A_{p, \alpha}+B_{p, \alpha}\right)-B_{p, \alpha}\right)\left[(s-p+1)^{1-\alpha}-(s-p)^{1-\alpha}\right]\right|}{|-4 a|}\right)<1 .
$$

\section{Numerical results}

We consider the following problem:

$$
{ }_{0}^{C P C} D_{x}^{\alpha} u(x)=\sin (x)
$$

where

$$
{ }_{0}^{C P C} D_{x}^{\alpha} u(x)=\frac{1}{\Gamma(1-\alpha)} \int_{0}^{x}\left(k_{1}(\alpha) u(t)+k_{0}(\alpha) \frac{d u(t)}{d t}\right)(x-t)^{-\alpha} d t
$$

We apply the Laplace transform to Eq. (5.1):

$$
L\left({ }_{0}^{C P C} D_{x}^{\alpha} u(x)\right)=L(\sin (x)) .
$$

Then we obtain

$$
\left[\frac{K_{1}(\alpha)}{s}+K_{0}(\alpha)\right] s^{\alpha} L(u(t))-K_{0}(\alpha) s^{\alpha-1} u(0)=\frac{1}{1+s^{2}}
$$

After simplification, we get

$$
L(u(x))=\frac{\left(1+s^{2}\right) K_{0}(\alpha) s^{\alpha-1} u(0)+1}{\left(1+s^{2}\right)\left(s^{\alpha-1} K_{1}(\alpha)+s^{\alpha} K_{0}(\alpha)\right)} .
$$

If we apply the inverse Laplace transform to the above equation, we will obtain

$$
u(x)=u(0) \exp \left(\frac{-K_{1}(\alpha)}{K_{0}(\alpha)} x\right)+\frac{x^{\alpha} A(x, \alpha)}{\left(K_{1}(\alpha)^{2}+K_{2}(\alpha)^{2}\right) \Gamma(\alpha)}
$$

where

$$
\begin{aligned}
A(x, \alpha)= & K_{1}(\alpha) \exp \left(\frac{-K_{1}(\alpha)}{K_{0}(\alpha)} x\right)\left(-\frac{K_{1}(\alpha)}{K_{0}(\alpha)}\right)^{-\alpha}\left(-\Gamma(\alpha)+\Gamma\left(\alpha, \frac{-K_{1}(\alpha)}{K_{1}(\alpha)} x\right)\right) \\
& +\frac{1}{\alpha} \text { HypergeometricPFQ }\left[\left\{\frac{\alpha}{2}\right\},\left\{\frac{1}{2}, 1+\frac{\alpha}{2}\right\},-\frac{x^{2}}{4}\right]
\end{aligned}
$$




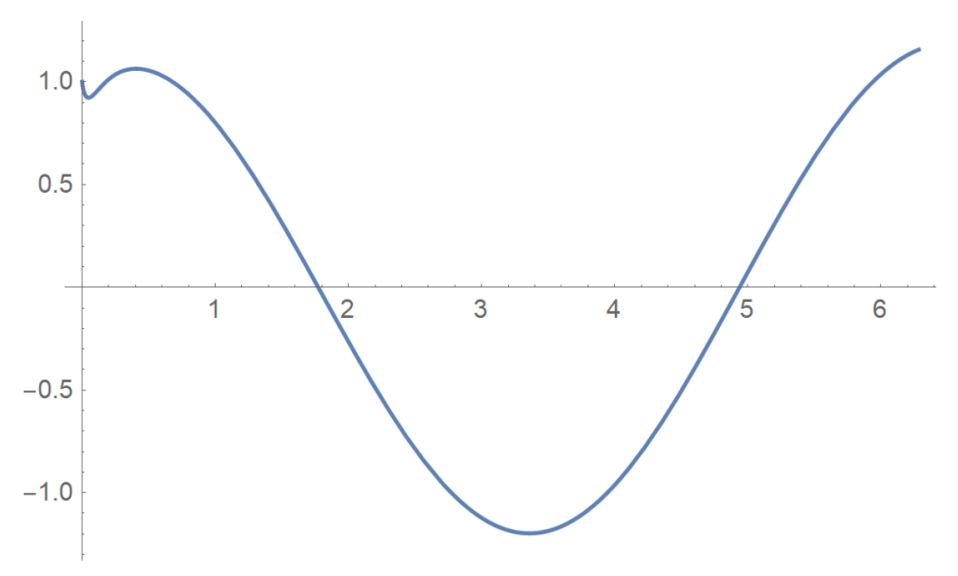

Figure 1 Solution of the problem for $\alpha=0.1$

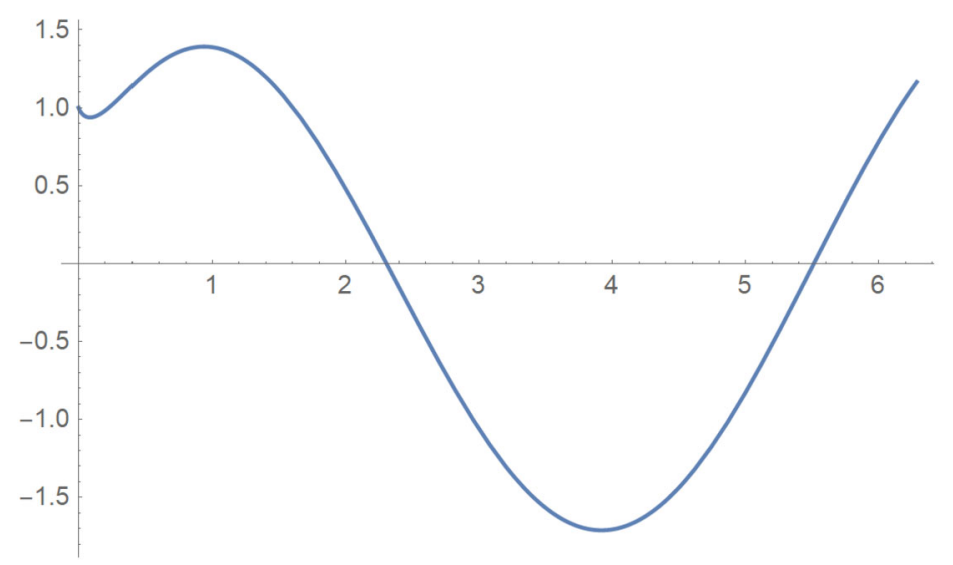

Figure 2 Solution of the problem for $\alpha=0.3$

$$
\begin{aligned}
& \times\left(K_{1}(\alpha) \cos (x)+K_{0}(\alpha) \sin (x)\right)+\frac{1}{1+\alpha} x\left(-K_{0}(\alpha) \cos (x)+K_{1}(\alpha) \sin (x)\right) \\
& \times \text { HypergeometricPFQ }\left[\left\{\frac{1}{2}+\frac{\alpha}{2}\right\},\left\{\frac{3}{2}, \frac{3}{2}, \frac{\alpha}{2}\right\},-\frac{x^{2}}{4}\right] .
\end{aligned}
$$

We demonstrate the above solution by the following figures for different values of $\alpha$. We choose $K_{1}(\alpha)=(1-\alpha) w^{\alpha}, K_{0}(\alpha)=\alpha c^{2 \alpha} w^{1-\alpha}, c=1, w=0.5$ and $u(0)=1$ in Figs. 1-6. In Fig. 7, we choose $c=w=\alpha=0.8$. In these figures, we can see the effect of the fractional order.

\section{Conclusion}

We presented the analysis of the proportional Caputo derivative in this paper. We presented some scientific theorems for this new derivative. We discretized the new derivative. We presented the stability analysis and experiments. We obtained the stability condition for a problem using the new derivative. We considered a problem with the constant proportional Caputo derivative. We solved the problem by the Laplace transform. We demonstrated the numerical simulations by some figures. 
Akgül and Baleanu Advances in Difference Equations

(2021) 2021:136

Page 10 of 12

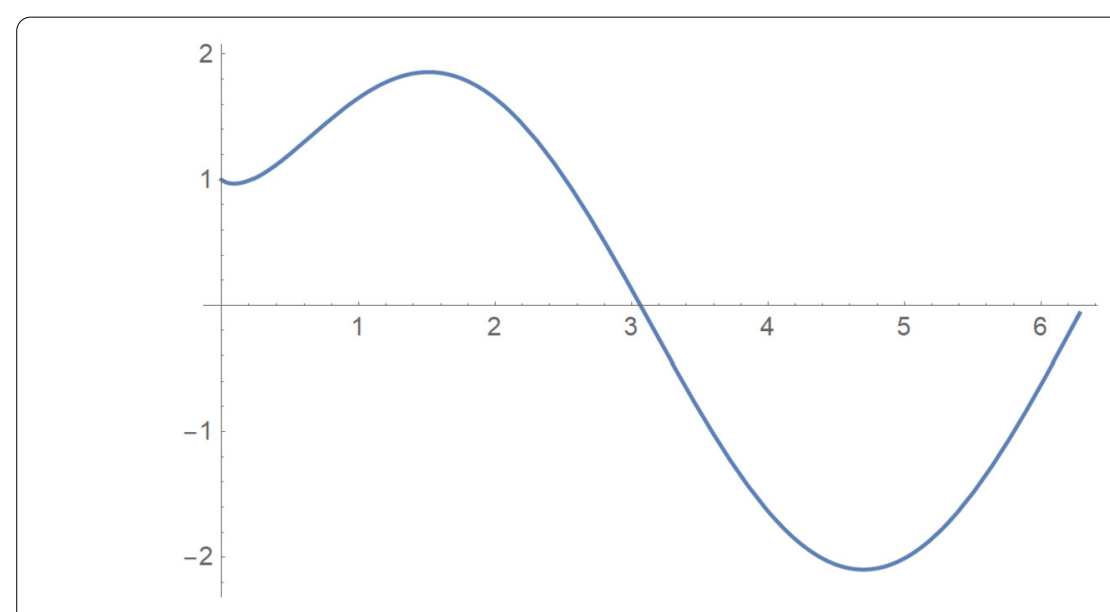

Figure 3 Solution of the problem for $\alpha=0.5$

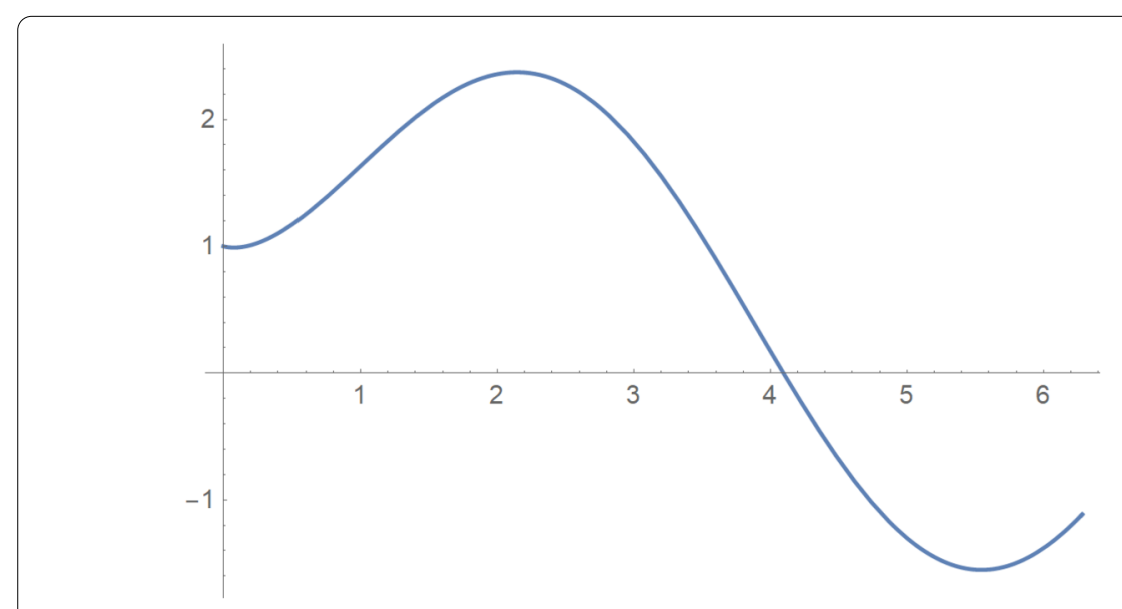

Figure 4 Solution of the problem for $\alpha=0.7$

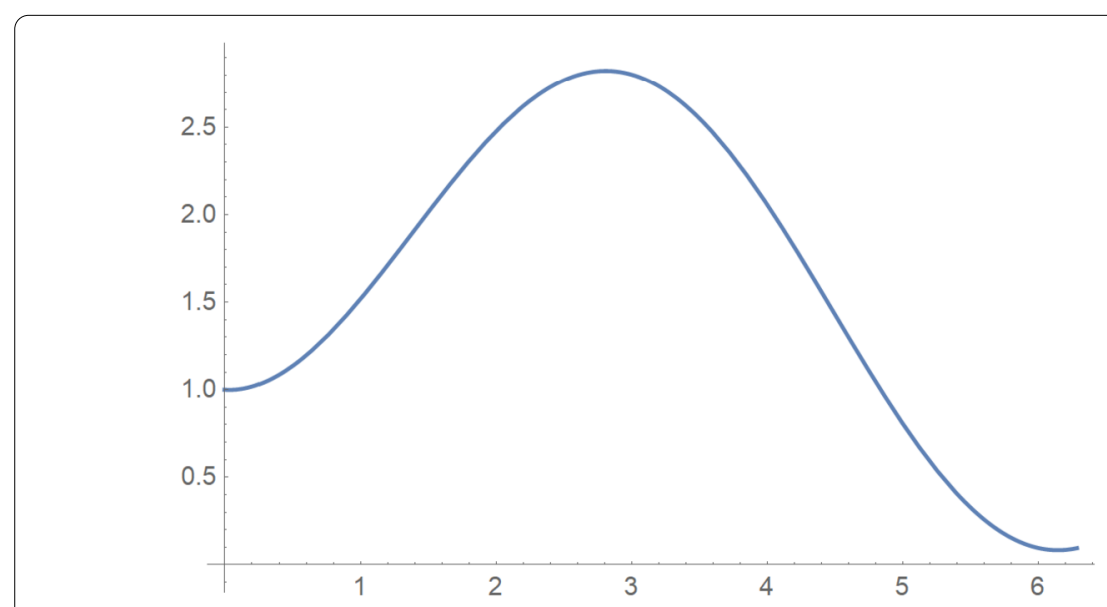

Figure 5 Solution of the problem for $\alpha=0.9$ 


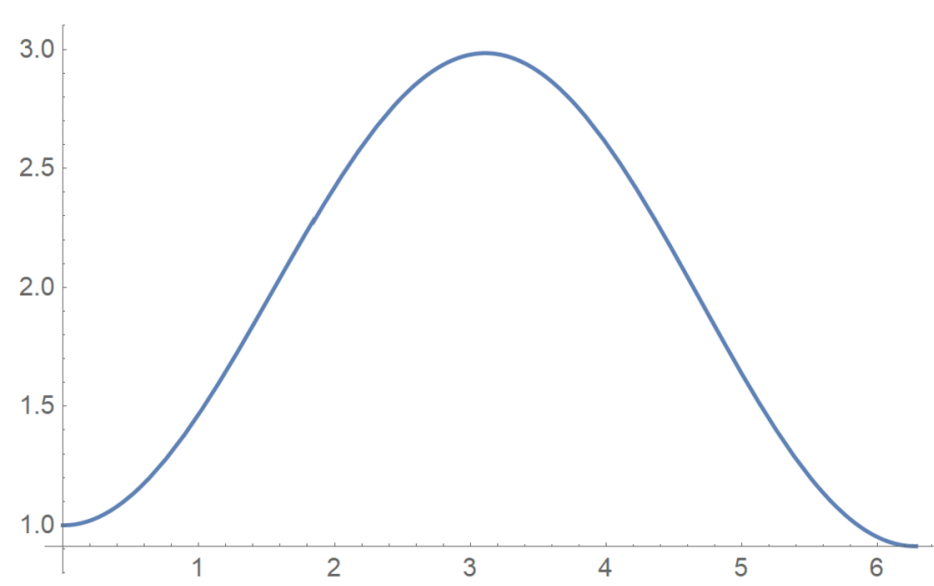

Figure 6 Solution of the problem for $\alpha=0.99$

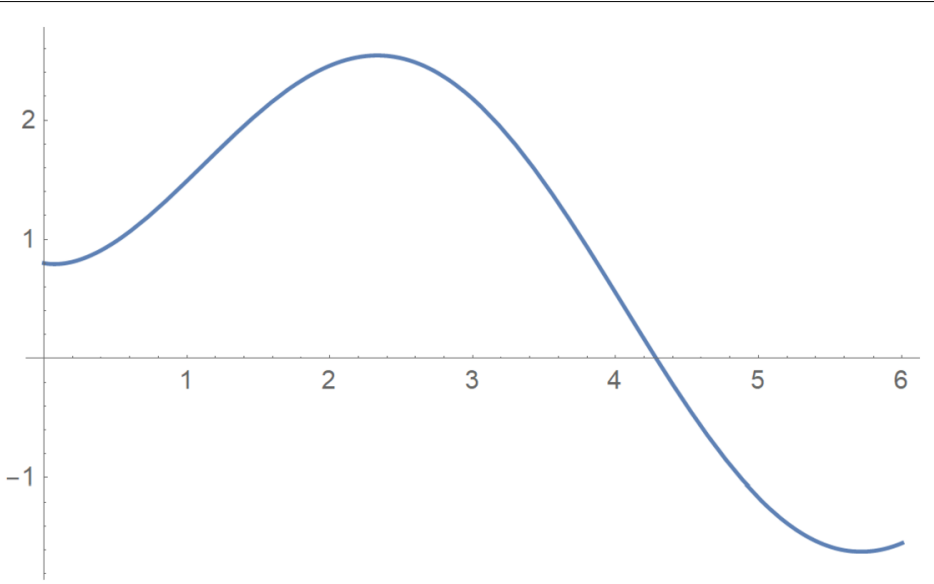

Figure 7 Solution of the problem for $\alpha=w=c=0.8$

\section{Acknowledgements}

We thank the reviewers for their comments to greatly improve the quality of our paper.

\section{Funding}

Not applicable.

\section{Availability of data and materials}

Not applicable.

\section{Competing interests}

The authors declare that they have no competing interests.

\section{Authors' contributions}

The authors have worked equally when writing this paper. All authors read and approved the final manuscript.

\section{Author details}

${ }^{1}$ Art and Science Faculty, Department of Mathematics, Siirt University, TR-56100 Siirt, Turkey. ${ }^{2}$ Department of Mathematics, Cankaya University, 06530 Balgat, Ankara, Turkey. ${ }^{3}$ Institute of Space Sciences, Magurele-Bucharest, Romania.

\section{Publisher's Note}

Springer Nature remains neutral with regard to jurisdictional claims in published maps and institutional affiliations.

Received: 15 May 2020 Accepted: 14 February 2021 Published online: 25 February 2021 
References

1. Kilbas, A.A., Srivastava, H.H., Trujillo, J.J.: Theory and Applications of Fractional Differential Equations. Elsevier, New York (2006)

2. Hilfer, R.: Applications of Fractional Calculus in Physics. World Scientific, Singapore (2000)

3. Baleanu, D., Diethelm, K., Scalas, E., Trujillo, J.J.: Fractional Calculus Models and Numerical Methods, Series on Complexity, Nonlinearity and Chaos, Frist edn. 2012/Second edn. 2016. World Scientific, Singapore (2016)

4. Baleanu, D., Etemad, S., Rezapour, S.: A hybrid Caputo fractional modeling for thermostat with hybrid boundary value conditions. Bound. Value Probl. 2020, 64 (2020)

5. Kumar, S., Baleanu, D.: A new numerical method for time fractional non-linear Sharma-Tasso-Oliver equation and Klein-Gordon equation with exponential kernel law. Front. Phys. 8, 136 (2020)

6. Ross, B.: A brief history and exposition of the fundamental theory of fractional calculus. In: Fractional Calculus and Its Applications. Lect. Notes Math., pp. 1-36. Springer, Berlin (1975)

7. Ortiqueira, M., Tenreiro Machado, J.A.: What is a fractional derivative? J. Comput. Phys. 293, 4-13 (2015)

8. Caputo, M., Fabrizio, M.: On the notion of fractional derivative and applications to the hysteresis phenomena. Meccanica 52(13), 3043-3052 (2017)

9. Zhao, D., Luo, M.: Representations of acting processes and memory effects: general fractional derivative and its application to theory of heat conduction with finite wave speeds. Appl. Math. Comput. 346, 531-544 (2019)

10. Hilfer, R., Luchko, Y.: Desiderata for fractional derivatives and integrals. Mathematics 7, 149 (2019). https://doi.org/10.3390/math7020149

11. Baleanu, D., Fernandez, A.: On fractional operators and their classifications. Mathematics 7, 830 (2019)

12. Caputo, M., Fabrizio, M.: A new defifinition of fractional derivative without singular kernel. Prog. Fract. Differ. Appl. 1(2), 73-85 (2015)

13. Atangana, A., Baleanu, D.: New fractional derivatives with nonlocal and non-singular kernel: theory and application to heat transfer model. Therm. Sci. 20(2), 763-769 (2016)

14. Khalid, N., Abbas, M., lqbal, M.K., Baleanu, D.: A numerical investigation of Caputo time fractional Allen-Cahn equation using redefined cubic B-spline functions. Adv. Differ. Equ. 2020, 158 (2020)

15. Owolabi, K.M.: Analysis and numerical simulation of multicomponent system with Atangana-Baleanu fractional derivative. Chaos Solitons Fractals 115, 127-134 (2018)

16. Akgül, A.: A novel method for a fractional derivative with non-local and non-singular kernel. Chaos Solitons Fractals 114, 478-482 (2018)

17. Akgül, E.K.: Solutions of the linear and nonlinear differential equations within the generalized fractional derivatives. Chaos 29, 023108 (2019)

18. Atangana, A., Akgül, A.: Analysis and Applications of the New Derivative, Chapter of Fractional Order Analysis: Theory, Methods and Applications. Wiley, New York (2020)

19. Fernandez, A., Baleanu, D., Srivastava, H.M.: Series representations for fractional-calculus operators involving generalised Mittag-Leffler functions. Commun. Nonlinear Sci. Numer. Simul. 67, 517-527 (2019)

20. Wu, G.C., Zeng, D.Q., Baleanu, D.: Fractional impulsive differential equations: exact solutions, integral equations and short memory case. Fract. Calc. Appl. Anal. 22, 180-192 (2019)

21. Rahman, G., Nisar, K.S., Abdeljawad, T.: Certain Hadamard proportional fractional integral inequalities. Mathematics 8(4), 504 (2020)

22. Jarad, F., Alqudah, M.A., Abdeljawad, T.: On more general forms of proportional fractional operators. Open Math. 18(1), 167-176 (2020)

23. Alzabut, J., Abdeljawad, T., Jarad, F., Sudsutad, W.: A Gronwall inequality via the generalized proportional fractional derivative with applications. J. Inequal. Appl. 2019, 101 (2019)

24. Baleanu, D., Fernandez, A., Akgül, A.: On a fractional operator combining proportional and classical differintegrals. Mathematics 8, $360(2020)$

25. Agarwal, P., Choi, J., Paris, R.B.: Extended Riemann-Liouville type fractional derivative operator with applications. Open Math. 15(1), 1667-1681 (2017)

26. Al-Sayed, A.A., Agarwal, P.: Numerical solution of multiterm variable-order fractional differential equations via shifted Legendre polynomials. Math. Methods Appl. Sci. 42(11), 3978-3991 (2019)

27. Agarwal, P., Rogosin, S.V., Trujillo, J.J.: Certain fractional integral operators and the generalized multi-index Mittag-Leffler functions. Proc. Math. Sci. 125(3), 291-306 (2015)

28. Cetinkaya, A., Kiymaz, I.O., Agarwal, P., Agarwal, R.: A comparative study on generating function relations for generalized hypergeometric functions via generalized fractional operators. Adv. Differ. Equ. 2018(1), 1 (2018)

\section{Submit your manuscript to a SpringerOpen ${ }^{\circ}$ journal and benefit from:}

- Convenient online submission

- Rigorous peer review

- Open access: articles freely available online

- High visibility within the field

Retaining the copyright to your article

Submit your next manuscript at $\boldsymbol{s p r i n g e r o p e n . c o m ~}$ 